\title{
Electronic excitations induced by the impact of coinage metal ions and clusters on a rare gas matrix: Neutralization and luminescence
}

\author{
Wolfgang Harbich, ${ }^{1}$ Christoph Sieber, ${ }^{1}$ Karl-Heinz Meiwes-Broer ${ }^{2}$ and Christian Félix ${ }^{1}$ \\ ${ }^{1}$ Institut de Physique des Nanostructures, École Polytechnique Fédérale de Lausanne (EPFL), CH-1015 Lausanne, Switzerland \\ ${ }^{2}$ Fachbereich Physik, Universität Rostock, D-18051 Rostock, Germany
}

(Received 14 June 2007; published 21 September 2007)

\begin{abstract}
Low-energy collisions of atomic ions and cluster ions with rare gas covered metal substrates can lead to strong light emission which is element and size specific. Instead of a direct energy transfer into the electronic system of the cluster the observed emission originates from excitons trapped in the rare gas layer which carries the excitation energy. The nature of the processes involved in the neutralization and excitation of the neutral clusters electronic system are discussed. Strong analogies to thermoluminescence are found.
\end{abstract}

DOI: 10.1103/PhysRevB.76.104306

PACS number(s): 61.46.Bc, 36.40.Vz, 36.40.Qv, 72.20.-i

\section{INTRODUCTION}

Size selected cluster deposition is a powerful alternative to kinetic controlled growth mechanisms ${ }^{1-11}$ for the production of model nanoscopic systems. The advantage of this approach is that it allows a precise tuning of the system size as well as a simultaneous control of the concentration. The deposition process necessarily consist of an ion-surface collision since the mass selection process requires the particle to be charged. The control on this process is crucial for insuring the integrity of the deposited clusters. Here we show that even on a very inert substrate, like a rare gas matrix, the deposition can lead to electronic excitations of the substrate.

Classical molecular dynamics calculations ${ }^{12-15}$ have extensively been used to describe the nuclear motion during the deposition. However, much less studies have focused on electronic processes. Ion surface collisions result in the excitation of the surface and particles nuclear and electronic system. Electronic excitations can lead to electron and photon emission or simply be absorbed by the substrate. ${ }^{16}$ In the first two cases, information may be gained on the particle substrate electronic system. ${ }^{17}$ More specifically in ion surface collisions charge transfer processes from the substrate to the ionized projectile become an important energy relaxation channel. In particular, in low-energy deposition, as will be described below, the charge transfer channel considerably contributes to the energy relaxation mechanism.

Rare gas matrices are used as an ideal support or spacer layer for a number of reasons: they are the insulators with the widest known band gap and are therefore transparent to light up to vacuum ultraviolet (VUV) energies; they are very inert and therefore do not react strongly with the deposited clusters (at least in the electronic ground state); they are soft and therefore adsorb the incident deposition energy, thus reducing the projectile fragmentation. ${ }^{4,12}$ Rare gas solids have also the interesting property that they are transparent to supplementary electrons injected in their conduction band, they are even more transparent to these electrons than to visible light. ${ }^{18}$ Under the influence of an electric field the conduction electrons are accelerated until they can release their energy by electronic excitations in the rare gas solid. ${ }^{18}$ When impurities are introduced in the rare gas matrix, luminescence of the impurities is observed due to energy exchange mechanisms. ${ }^{19}$ Since small metal clusters have been shown to exhibit fluorescence, ${ }^{7,20-22}$ they should therefore be excited by such an exchange mechanism.

In this paper we report the observation of luminescence during the impact of ions and cluster ions on rare gas matrices. This is an extension of the experimental evidences and a refinement of the model discussed in Ref. 23. The luminescence and the neutralization of the ions resulting from the deposition process are investigated. Insights in the electronic processes involved in the deposition are gained. We discuss first the origin of the energy necessary for the neutralization of the incoming ions and later the mechanisms leading to the observed luminescence. Finally, we propose a microscopic model that is consistent with the experimental evidences and is, incidentally, perfectly compatible with the mechanisms invoked in the thermostimulated phenomena mentioned above.

\section{EXPERIMENTAL SETUP}

The experimental setup is described only briefly, a more complete description has been given elsewhere. ${ }^{24}$ Metal clusters are produced by sputtering from a metal target using an intense and high-energy $\mathrm{Xe}^{+}$beam (typically $10 \mathrm{~mA}$, $24 \mathrm{keV}$ ). They are extracted by an ion lens system and mass selected by a quadrupole mass filter. The mass selected cluster ions are deflected by $90^{\circ}$ with a quadrupole, that acts as a filter for the neutral silver particles as well as an energy filter for the cluster ions. The positively charged clusters are focused by a second lens system on a cold $(<25 \mathrm{~K})$ gold plated copper or graphite (HOPG) substrate (see Fig. 1), where they are codeposited with the Ar to form a seeded matrix. No external neutralization electrons are provided to the ions. The sample is electrically isolated and by setting its potential, the deposition energy of the clusters can be varied and the deposition current measured. The deposition energy ranges from a few $\mathrm{eV}$ to several tens $\mathrm{eV}$, with a particle current of a few nA. The potential between the last lens (LL) and the support can be varied to control the flow of electrons leaving the matrix (see discussion on the neutralization process).

The light is collected during the deposition by an optical fiber $(\phi=400 \mu \mathrm{m})$ located close to the cluster deposition 


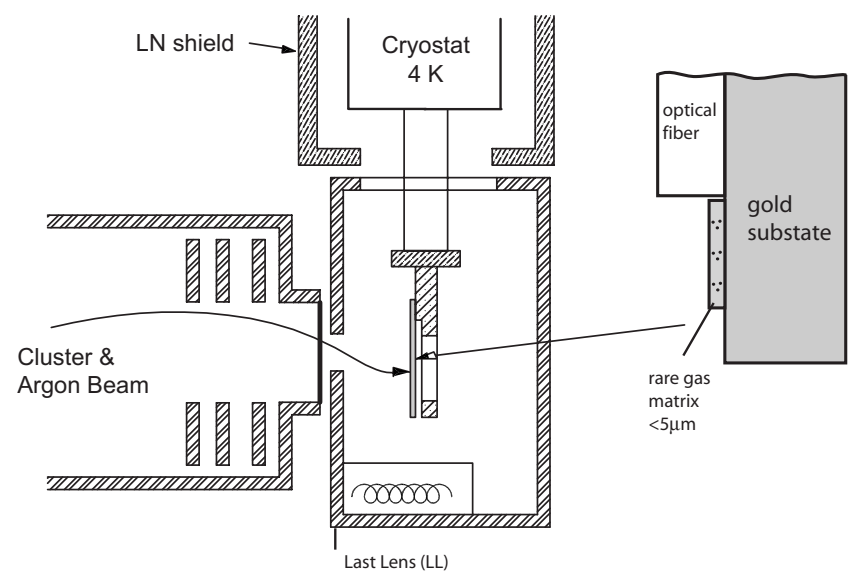

FIG. 1. Schematics of the deposition region. Inset: Light collection with an optical fiber placed in the vicinity of the deposition spot.

spot as shown in the inset of Fig. 1. This simple design is very small with a well-defined and rigid optical setup. A draw back, however, is the low detection efficiency of $\sim 5$ $\times 10^{-4}$. This is an order of magnitude less than what can be obtained by an efficient conventional optical system, but the collection optics is rigid and stable, it does not require any focal adjustment. The emitted light is analyzed by an optical spectrometer (Jobin-Yvon T64000) coupled to a liquid nitrogen CCD detector for spectra acquisitions. Alternatively a photomultiplier tube can be used for time resolved measurements. The spectra are not corrected for the transmission and efficiency of the different optical elements (optical fiber, spectrometer, CCD). The rare gas matrices are very dilute, the argon flux towards the cold surface is set in order to achieve an Ar:cluster ratio of typically $10^{4}: 1$.

\section{NEUTRALIZATION}

During cluster deposition the ion current is monitored continuously. While this is straightforward on the bare substrate, the rare gas covered surface needs some reflection. Figure 2 shows the ion current $I_{n}$ as a function of matrix thickness that, at constant gas flow rate, is equivalent to time. $I_{n}$ is measured by connecting the conductive substrate to the ground via a biasing power supply. Strictly speaking, $I_{n}$ measures the number of electrons that flow towards the sample to neutralize or compensate the positive charges of the clusters. The capacitive current, where the electrons accumulate at the metal-matrix interface and compensate the positive charges in the matrix contributes only partially to $I_{n}$. The accumulated charge and the corresponding potential build up would prevent further clusters from arriving after fairly short time. With the conditions of the measurements in Fig. 2 [current density, matrix growth rate $(3 \AA / s)$ and deposition energy $(30 \mathrm{~V})]$, the space charge would build up in about $100 \mathrm{~s}$ or equivalently in a matrix thickness of $14 \mathrm{~nm}$. It is clear from the current reading (Fig. 2) that this does not happen here. Therefore the electrons have to flow into the matrix and neutralize the cluster ions. This conclusion is furthermore sup-

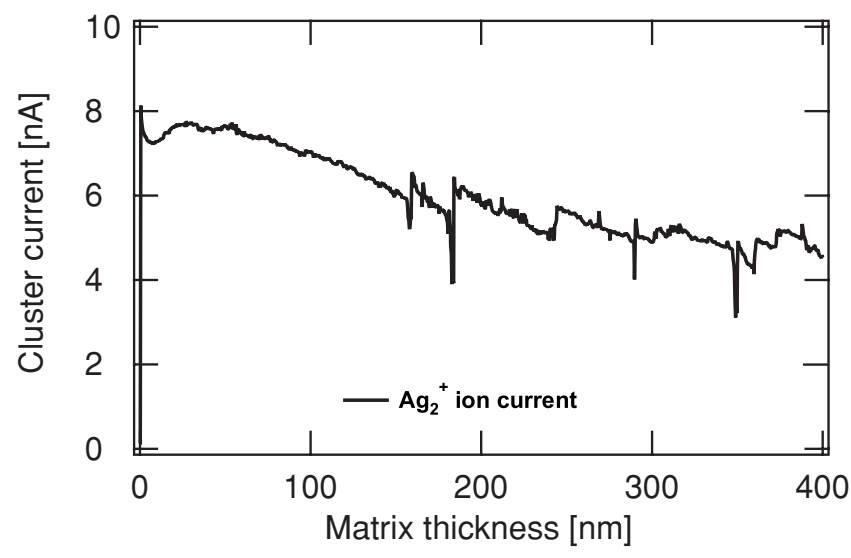

FIG. 2. Evolution of the $\mathrm{Ag}_{2}^{+}$current as a function of matrix thickness measured at a temperature of $20 \mathrm{~K}$. Since Ar is dosed at a constant rate of $0.3 \mathrm{~nm} / \mathrm{s}$ the maximum thickness corresponds to a time of $1200 \mathrm{~s}$.

ported by the fact that, as discussed below, we observe the optical spectra of neutral species. To rule out the possibility that the electrons flow on the surface of the matrix and not through the matrix, we have repeated the experiments by building the matrix from a static argon pressure in the deposition chamber and not from a directed flow. This means that all cold surfaces are covered by an argon matrix simultaneously. No significant changes have been observed proving that electrons flow indeed from the metal surface into the gas matrix towards the cluster ions.

It is well known that excess electrons in rare gas matrices are almost unbound and can move very easily through a liquid or a solid rare gas ${ }^{18}$ until they find either a structural defect that can trap them or an ion that they can neutralize. By setting the potentials surrounding the matrix attractive for the electrons (see Fig. 1), they start to flow out of the matrix, because argon has a conduction band that is above the vacuum level $^{25}$ (see Fig 5). The dependence of the current reading with the potential difference between the substrate and the surrounding lenses is illustrated in Fig. 3. Very small changes in the electric field outside the matrix which is achieved by varying the potential on lens LL show signifi-

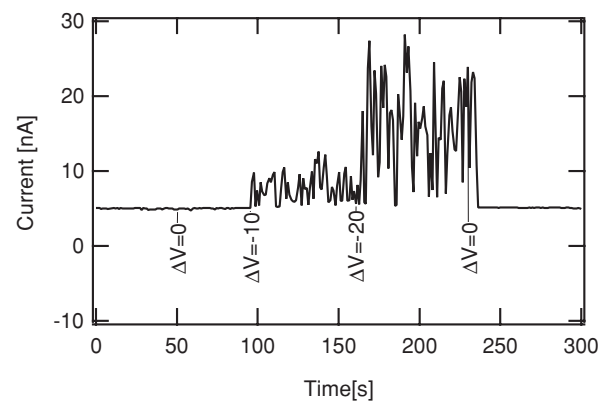

FIG. 3. Current reading versus the potential difference $\Delta V$ between the sample holder and the last lens element before the sample holder (the distance between the two planes is $\sim 5 \mathrm{~mm}$ ). Note that the fluctuations in the current increase dramatically even for very small potential difference. See text for details. 


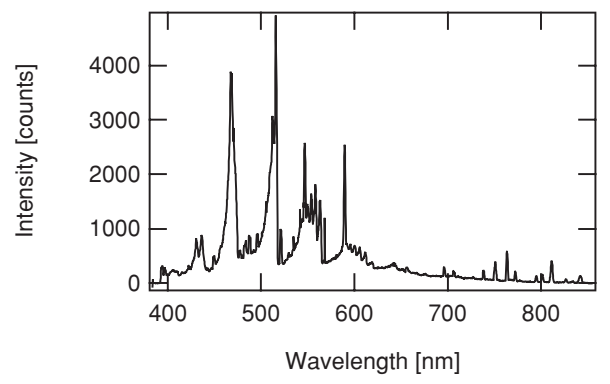

FIG. 4. Spectral signature of the argon discharges. See text for details.

cant effects on the measured neutralization current $I_{n}$. We observe a strong increase (about a factor of 3 at $20 \mathrm{~V}$ ) and $I_{n}$ becomes instable. Up to two orders of magnitude higher $I_{n}$ currents have been measured than the actual cluster current.

Very moderate external electrical fields allow electrons to leave the matrix and therefore favor an accumulation of positive charges in the matrix; the maximal achievable potential corresponds to the deposition energy and can be reached over very short distances (typically $20 \mathrm{~nm}$ ) if the clusters are not neutralized. ${ }^{58}$ This yields very intense electrical fields in the matrix. Above $50 \mathrm{eV}$ deposition energy, these strong fields are evidenced by discharges that eventually destroy the matrix. The discharges in argon can be monitored via the intense optical signature reported in Fig. 4. It is important to mention that for all electric fields reported $I_{n}$ drops to 0 when the incoming cluster current $I_{c}$ is 0 .

The first question to answer is the mechanism for the production of the "free" electrons in the rare gas solid. The energy diagram of the system is sketched in Fig. 5. It ignores structural defects or impurities that add states in the band gap of argon. Conduction electrons from the conducting substrate
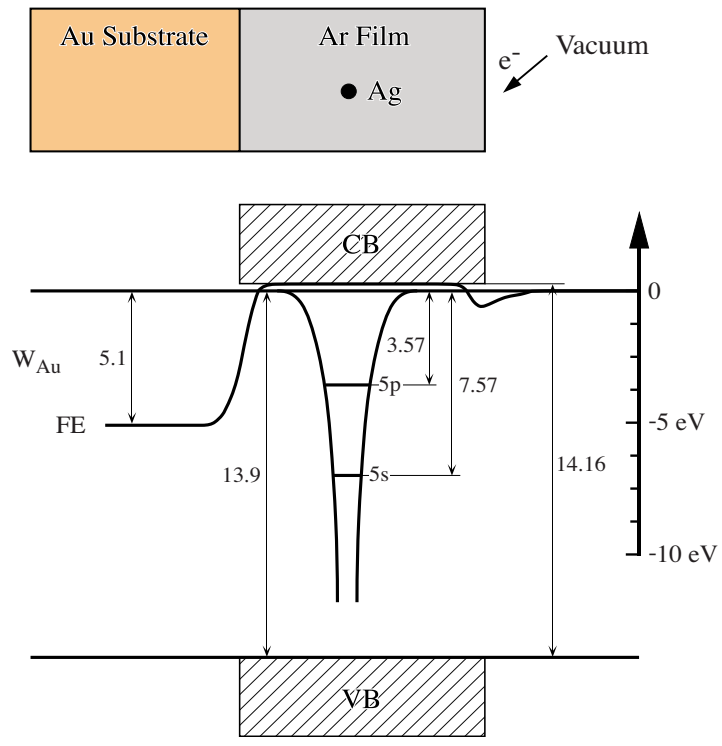

FIG. 5. (Color online) This diagram sketches the energetics of the studied system in the absence of external electric fields. The system is considered as ideal, i.e., without structural defects or impurities. See text for details. have to first overcome a barrier of more than $5 \mathrm{eV}$ [work function of the gold substrate $W_{\text {Au }}$ plus the small energy which separates the vacuum level (VL) from the bottom of the conduction band (CB) of argon]. Once in the matrix the excess electrons can move almost freely. There are only a few possible mechanisms that allow the electrons to overcome this barrier. Field emission is in principle possible as the local electric field due to the accumulation of ions can be very strong. For example if the clusters are deposited with an energy of $30 \mathrm{eV}$, the accumulated charges can build a potential up to $30 \mathrm{~V}$ (after that all the incoming charged clusters will be deflected). This happens on very short distances $\sim 20 \mathrm{~nm}$ resulting in an electrical field of $\sim 10^{9} \mathrm{~V} / \mathrm{m}$, largely sufficient to allow for field emission. The efficiency of such a mechanism would however depend exponentially on the distance. Instead the measurements reported in Figs. 2 and in 6(c), for example, cannot be explained by the FowlerNordheim equation, which describes the field emission mechanism.

The impact energy of the cluster is sufficient to overcome the energy barrier provided that there is a mechanism to transfer the kinetic energy into an electronic excitation and a way to transfer this energy from the matrix surface to the metal-matrix interface. Several reviews of electronic processes in rare gas solids exist, ${ }^{25-28}$ we refer to them for the details of the relaxation mechanisms discussed hereafter. An excitation above the energy gap results in electron-hole pair (free exciton) formation. In the recombination of an exciton a VUV photon is emitted. ${ }^{25,28}$ Given the geometry the VUV photon has $\sim 50 \%$ probability to be captured by the surface and to induce a photoelectron into the conduction band of the rare gas (see Fig. 15 for a schematic view of the mechanism).

We are not aware of experiments reporting the production of excitons by low-energy ion impact on a rare gas surface, however, there are indications that this is a possible process. Sputtering of atoms and molecules from the surfaces of rare gas solids upon excitation by energetic electron $(\mathrm{keV})$ or ion beams ( $\mathrm{keV}$ to $\mathrm{MeV}$ ) has been studied by different groups; a review on this subject can be found in Ref. 29. The sputtering yield is high even for the small binding energies of the ejected species at the surface $(60 \mathrm{meV}$ in $\mathrm{Ar})$. From these observations it has been concluded that the mechanism for the ejection result from electronic excitation. More specifically the high kinetic energy of the sputtered rare gas atoms was interpreted as arising from the vibrational energy associated with the decay of molecular self-trapped excitons near the rare gas matrix surface ${ }^{30}$ At these high collision energies the production of excitons by the impact of ions has been observed, unfortunately these experiments have not been extended to lower energies.

Precursors of free excitons, namely, metastable argon atoms, have been reported in atomic collisions with rare gas atoms. Visible and UV light emission were observed in the case of $\mathrm{H}$ atom or ion collisions with rare gas atoms ${ }^{31-34}$ and this even at energies as low as $\sim 10 \mathrm{eV}$. Metastable atoms and VUV emission have been observed in collisions between rare gas ions and atoms as well as between two neutral rare gas atoms. ${ }^{35-37}$ Ionization has been reported in the case of collisions of two neutral rare gas atoms at low collision energies. $^{38}$ 

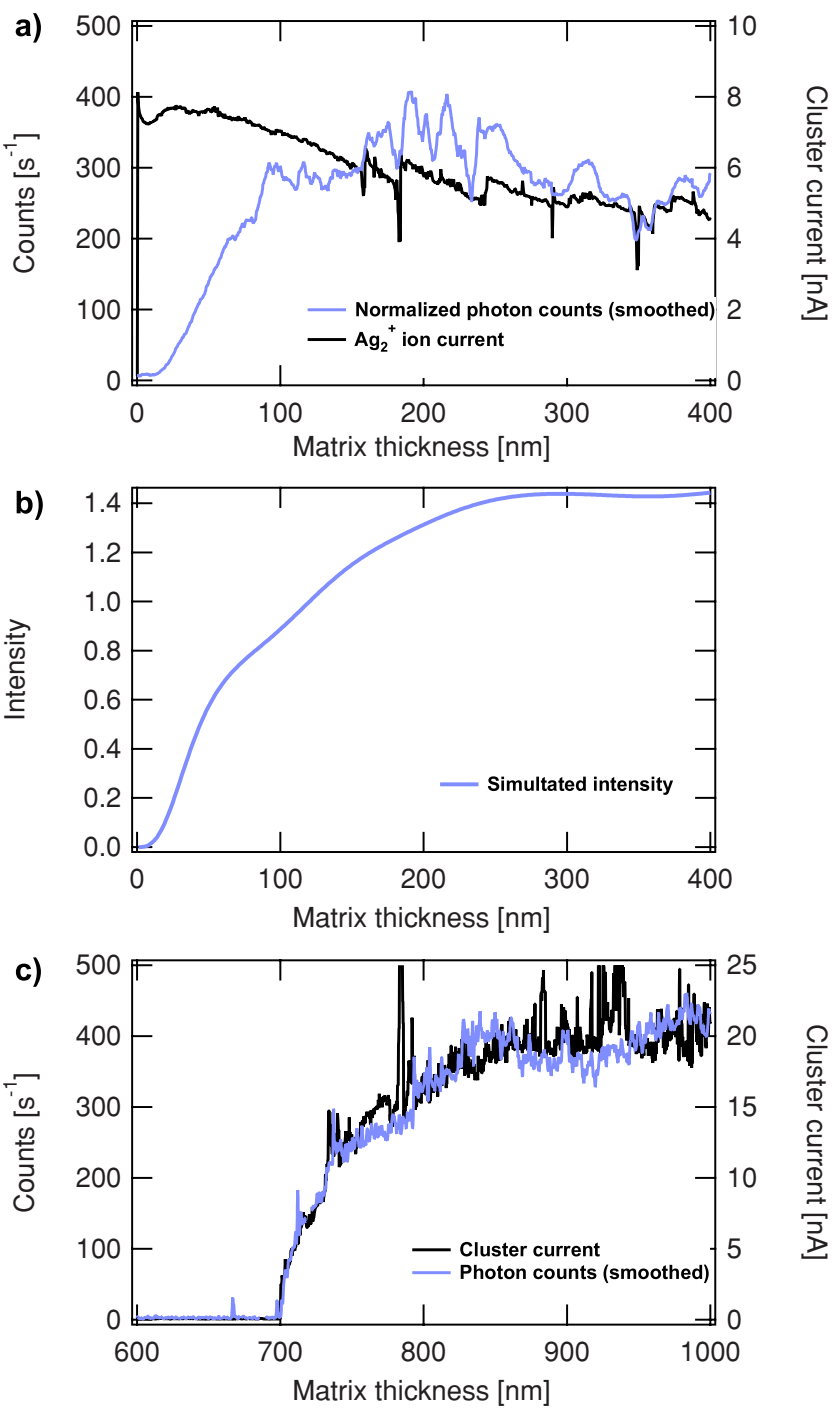

FIG. 6. (Color online) Evolution of light emitted by $\mathrm{Ag}_{2}^{+}$collisions measured at a matrix temperature of $20 \mathrm{~K}$. (a) The deposition starts with no argon matrix on the metal surface. The clusters are deposited together with argon to form a seeded matrix. No light emission is observed at zero coverage, the luminescence starts with a matrix thickness of $\sim 20 \mathrm{~nm}$ and saturates after $\sim 200 \mathrm{~nm}$. The deposited current reading is identical to the one of Fig. 2 and the photon counts have been corrected for current fluctuations. (b) Simulated intensity of the emission at $470 \mathrm{~nm}$ parallel to a gold surface (see text). (c) The matrix is first built without clusters until a thickness of $700 \mathrm{~nm}$ and then the clusters are directed towards the matrix together with argon to form a seeded matrix. In this case the emitted light is directly proportional to the cluster current. For technical reasons, the cluster current is slowly increased over time.

So, on the one hand, it is known that even low collision energies can strongly excite the electronic system of charged or neutral atoms. Given the reports mentioned above and our experimental evidences, we conclude that free excitons are also produced during the impact of low-energy ions on rare gas solids. ${ }^{59}$

Once the photoelectron produced it moves almost freely in the conduction band $(\mathrm{CB})$ of the rare gas solid. It can be accelerated by the intrinsic electric fields built up by the incoming charges, the speed of the electrons is limited by inelastic collisions accompanied with electronic excitations in the rare gas. ${ }^{18}$ The maximum acceleration voltage $V_{\max }$ for the electrons is limited by the deposition energy of the clusters $E_{\text {dep }}$, which sets the upper limit for the potential difference. When $E_{\text {dep }}$ exceeds the exciton energy, which is the case here, the accelerated electron can produce a new exciton. This leads to an avalanche effect or the multiplication of electrons. This multiplication mechanism, first demonstrated by Usenko et al., ${ }^{19}$ explains the high neutralizing current shown in Fig. 3.

It should be noted here that the neutralization current does not depend on the crystallinity of the rare gas solid. This point is important in the forthcoming discussion on luminescence effects.

\section{STUDY OF THE LUMINESCENCE}

The discussion above has evidenced the excitation of the electronic system of the cluster-matrix-substrate unit. Apart from the production of photoelectrons from the metal surface, energy transfer to the metal clusters can lead to emission of photons. After a description of the observed phenomena, we extend the discussion of the exciton production to interpret the obtained results. This luminescence provides a unique opportunity to study the electronic processes occurring in the collision.

When clusters are directed on a bare metal substrate, no luminescence is observed. This is expected following the arguments of Lorente et al.: ${ }^{17}$ the total photon yield for a cluster ion impinging at an energy of $10 \mathrm{eV}$ on a metal substrate amounts to $\sim 5 \times 10^{-9}$, which is below our detection sensitivity. The reason for this low yield is essentially the very effective energy transfer mechanism for a radiative dipole in the vicinity of a metallic substrate. When a sufficiently thick matrix is deposited on the metal surface, a luminescence signal appears. Figure 6(a) shows the integrated photon intensity as a function of cluster ion current and matrix thickness. Note that in Fig. 6(a) light emission is delayed with respect to the cluster current because the matrix is built at the same time as deposition starts, while in Fig. 6(c) the photon intensity follows closely the current measurement. In that case a rare gas layer has been preformed before cluster ions are directed to the surface. The luminescence is therefore strongly linked to the presence of the rare gas matrix.

Interestingly the light emission only starts when the matrix reaches a thickness of $\sim 20 \mathrm{~nm}$. This indicates either that the luminescence can only occur with a matrix that is thick enough or the quenching of the luminescence due to the presence of a metal surface. When a thick matrix is grown first then the luminescence starts immediately and is linear with the incident cluster current as shown in Fig. 6(c).

It is well known ${ }^{39}$ that the presence of a metal surface influences the luminescence by the simple fact that a dipole interacts with its own radiation reflected by the surface. The problem has been studied, for example, by Chance et al., ${ }^{40}$ where they were interested in the lifetime of an excited molecule near a partially reflecting metal mirror. The signal intensity depends on the orientation of the dipole relative to the 


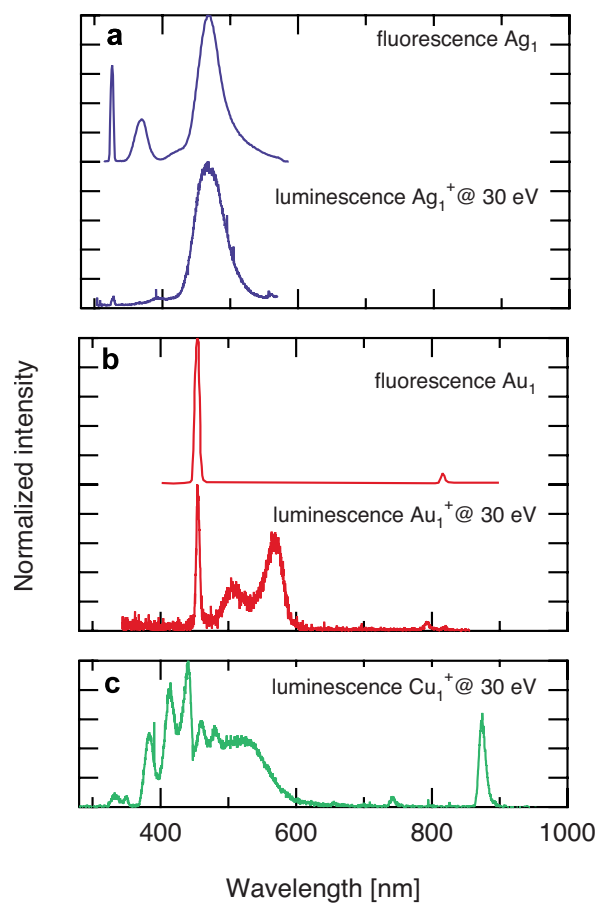

FIG. 7. (Color online) (a), (b) Spectrally resolved luminescence from $\mathrm{Ag}_{1}^{+}$and $\mathrm{Au}_{1}^{+}$collisions and laser induced fluorescence spectra of $\mathrm{Ag}_{1}$ and $\mathrm{Au}_{1}$ in argon for comparison. (c) Luminescence spectrum from $\mathrm{Cu}^{+}$collisions.

metal surface, on the distance to the surface, on the dielectric functions of the metal and matrix, and on the emission wavelength. Following Chance $e t$ al. we calculate the observed emission yield in front of a perfect gold surface. Since in our measurements the light is captured by an optical fiber placed parallel to the metal surface we need to average the calculated signals with dipoles parallel and perpendicular to the surface. The result of the calculation for an emission wavelength of $470 \mathrm{~nm}$, corresponding to the main emission for $\mathrm{Ag}_{2}^{+}$(see Fig. 8 for a complete spectrum) is shown in Fig. 6(b). The agreement between this simulation and the measurements shown in Fig. 6(a) is remarkable (there is no adjustable parameter) and fully explains the evolution of the luminescence intensity with the matrix thickness.

The analysis of the emitted light reveals its origin (Fig. 7). The spectral signature of the luminescence resulting from the impact of $\mathrm{Ag}_{1}^{+}, \mathrm{Au}_{1}^{+}$, and $\mathrm{Cu}_{1}^{+}$in argon is compared to optically excited fluorescence measurements performed on the corresponding neutral system; remember that for fluorescence measurements mass-selected clusters have been deposited with an excess of low energy electrons that allow for the cluster neutralization. ${ }^{41}$

For $\mathrm{Ag}_{1}^{+}$[Fig. 7(a)], the most intense peak of the luminescence and fluorescence at $465 \mathrm{~nm}$ are in excellent agreement and a second peak at $326 \mathrm{~nm}$ is also easily recognizable in both spectra although their relative intensities vary strongly. The fluorescence peak at $366 \mathrm{~nm}$ appears to be shifted to the red in the luminescence. Similarly for $\mathrm{Au}_{1}^{+}[$Fig. 7(b)] the narrow fluorescence peak at $454 \mathrm{~nm}$, as well as a less intense peak at $815 \mathrm{~nm}$ are present both in the luminescence due to collisions of ions and in the optically excited fluorescence of

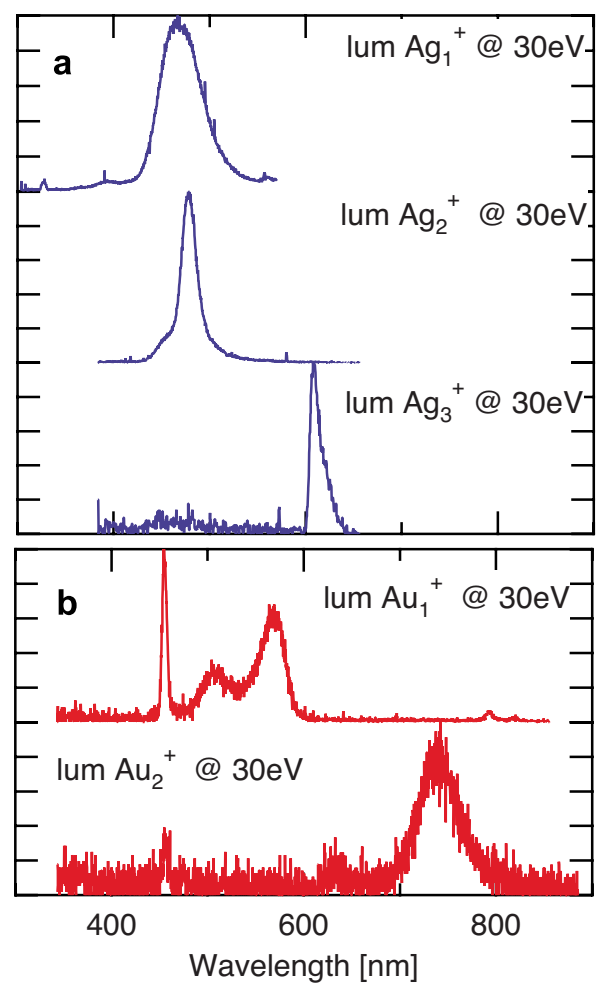

FIG. 8. (Color online) (a) Spectrally resolved luminescence of $\mathrm{Ag}_{1}^{+}, \mathrm{Ag}_{2}^{+}$, and $\mathrm{Ag}_{3}^{+}$during the deposition in an argon matrix. (b) Corresponding luminescence of $\mathrm{Au}_{1}^{+}$and $\mathrm{Au}_{2}^{+}$.

neutral $\mathrm{Au}$ atoms. The peak at $815 \mathrm{~nm}$ is proportionally less intense in the luminescence. Additional spectral features are present in the luminescence but do not show up in the fluorescence of the neutral specie in argon. ${ }^{20}$ Finally the luminescence of $\mathrm{Cu}_{1}^{+}$[Fig. 7(c)] shows peaks at 741 and $874 \mathrm{~nm}$ which correspond to the fluorescence peaks of $\mathrm{Cu}_{1}$ when excited at $311 \mathrm{~nm}$ (Ref. 42) as well as additional features.

The clear distinction between the different luminescence spectra and, moreover, identical positions of the peaks when compared to the optically excited fluorescence of the corresponding neutral particles embedded in argon proves that this luminescence originates from the neutralized particle. The luminescence of the neutral atom is observed despite the fact that the cation is deposited. It is therefore clear that either the fluorescence happens after the neutralization process or that it is a direct result of the neutralization, where the neutralizing electron radiates down to the ground state.

Differences in the spectral signatures are not surprising since the luminescence intensity depends on the excitation mechanism and the relaxation path to the state from which the luminescence occurs. Here the different excitation mechanism may populate states that could not be accessed with photons due to optical selection rules.

Next we consider the role of the size of the projectile. Figure 8(a) shows the light emitted during the deposition of silver ions and clusters $(n=1,2,3)$. The luminescence is in good agreement with the reported optically excited fluorescence spectra in argon. ${ }^{20,43}$ In the case of silver, light emission is restricted to the small systems. We could not observe any luminescence for larger particles, whereas laser induced 


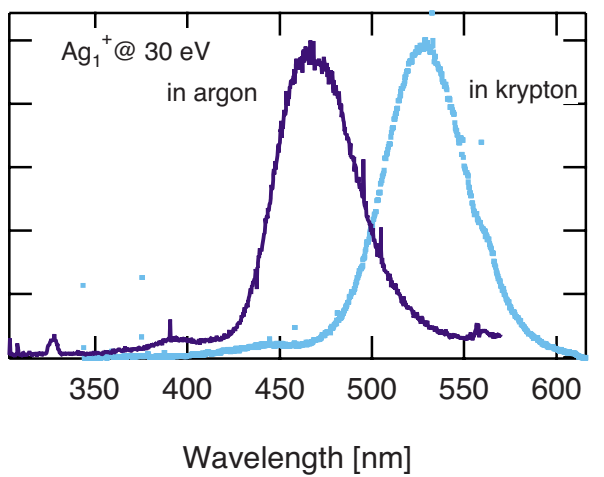

FIG. 9. (Color online) Spectrally resolved luminescence of $\mathrm{Ag}_{1}^{+}$, during the deposition in an argon and a krypton matrix.

fluorescence has been observed for $\mathrm{Ag}_{4},{ }^{21} \mathrm{Ag}_{8},{ }^{7}$ and $\mathrm{Ag}_{9}{ }^{22}$ Likewise the luminescence of gold monomer and dimer ions is reported on Fig. 8(b), it is again in good agreement with the known fluorescence spectra. ${ }^{20}$

As for the fluorescence, the luminescence depends on the host matrix. This is illustrated in Fig. 9 where $\mathrm{Ag}_{1}^{+}$colliding with argon and krypton are compared. The same matrix shifts as in fluorescence measurements ${ }^{44}$ are observed and this agreement hints at that the emitting particle has sufficient time to find a stable environment.

By changing the impact energy of the projectiles, the spectral response changes due to the fragmentation of the colliding particles as shown in Fig. 10. $\mathrm{Au}_{2}^{+}$is deposited in an argon matrix with three different impact energies $(30,100$, and $300 \mathrm{eV}$ ), the luminescence signal is resolved spectrally. For $30 \mathrm{eV}$ the dominant feature in the spectrum is the fluo-

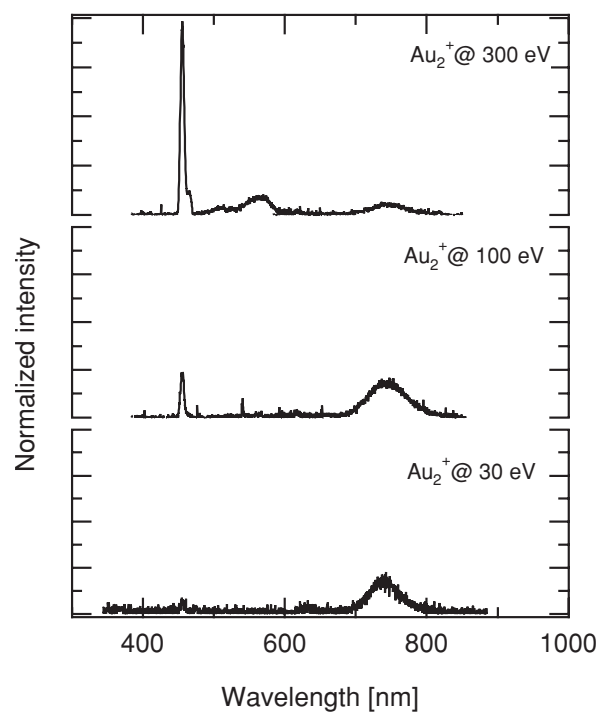

FIG. 10. Spectrally resolved luminescence of $\mathrm{Au}_{2}^{+}$, during the deposition into an argon matrix. The comparison with Fig. 8 shows that when the impact energy is raised the gold dimer fragments. The fact that we observe the luminescence of the fragment means that the luminescence process happens after the fragmentation has taken place. The spectra are normalized in order to keep the area below them constant.

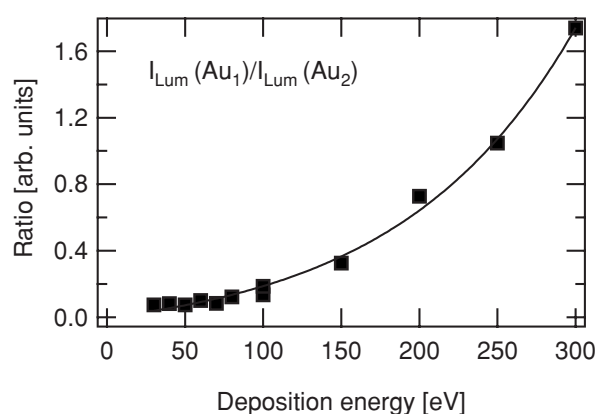

FIG. 11. Ratio between the intensity of the main peak associated with the luminescence of $\mathrm{Au}_{2}(745 \mathrm{~nm})$ and the main peak associated with the luminescence of $\mathrm{Au}_{1}(465 \mathrm{~nm})$ for different deposition energies. The line is a guide to the eye. The total current varies with the deposition energy. It is, however, impossible to normalize the signal with the deposition current, because of the electron avalanche process discussed above and illustrated in Fig. 3.

rescence signature of $\mathrm{Au}_{2}$, by increasing the deposition energy the dominant feature is changed to the fluorescence signature of $\mathrm{Au}_{1}$. These observations reinforce the previous statement and prove that the observed luminescence does not happen during but after the impact, for the cluster has the time to fragment and to capture an electron before emitting a photon.

Moreover we now have direct access to the fragmentation of the clusters as function of their impact energy. Figure 11 shows the ratio between the luminescence intensity for $\mathrm{Au}_{2}$ and for $\mathrm{Au}_{1}$ as a function of the deposition energy.

\section{ANALYSIS OF THE LUMINESCENCE PROCESS}

As already mentioned, light is observed only when the matrix is thick enough (Fig. 6). Moreover and a key observation in the following discussion, luminescence depends strongly on the quality of the rare gas matrix. Figure 12 demonstrates that the impacts of the clusters induce an annealing of the matrix associated with luminescence of the deposited cluster. A fresh matrix is deposited for $30 \mathrm{~s}$ at a temperature of $18 \mathrm{~K}$ while the cluster beam is stopped by switching off the quadrupole mass filter; the argon flow is then stopped and the clusters are allowed to reach the surface. The luminescence sets on immediately without any measurable delay and then it decreases exponentially with time showing that: the impacts of the clusters anneal the matrix locally, and the annealed matrix does not luminesce anymore. Growing a thicker matrix by waiting $60 \mathrm{~s}$ rather than $30 \mathrm{~s}$, the luminescence increases; further increasing of the matrix thickness does not increase the luminescence anymore, this translates in an active layer for luminescence of about $\sim 20 \mathrm{~nm}$. The luminescence process happens close to the matrix/vacuum interface, within these first $20 \mathrm{~nm}$, the rest of the matrix has no or much less influence. A cross section per impact of $\sim 320 \AA^{2}$ (at $30 \mathrm{eV}$ ) associated with the local annealing can be estimated from the dependence of the luminescence with coverage. A further proof that the luminescence is related to the imperfection of the matrix is given by its temperature dependence. The luminescence in- 

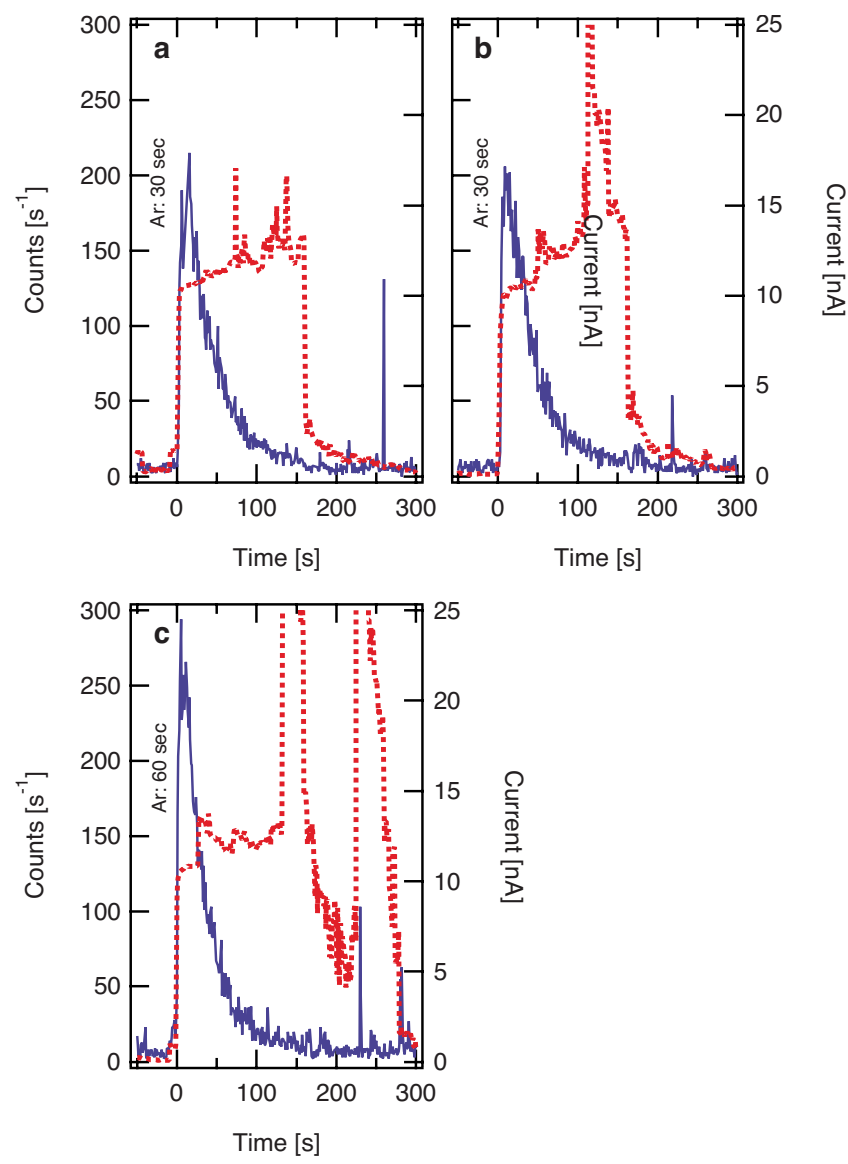

FIG. 12. (Color online) Luminescence intensity of $\mathrm{Ag}_{2}^{+}\left(E_{\text {dep }}\right.$ $=30 \mathrm{eV})$ versus time. The continuous line shows the luminescence and the dotted line the measured current. Three experiments are shown: a fresh matrix is grown at $18 \mathrm{~K}$ with no cluster current for the first $30 \mathrm{~s}$ [(a) and (b)] and for $60 \mathrm{~s}$ (c). After the "preparation" the cluster beam is switched on at $t=0$.

tensity depends strongly and smoothly on the matrix temperature as illustrated in Fig. 13. Clearly the structure of the matrix changes with the deposition temperature. ${ }^{45}$ According to Fugol' ${ }^{46}$ the size of the crystallites varies significantly with the condensation temperature at which the matrix is grown. When the condensation temperature is greater than

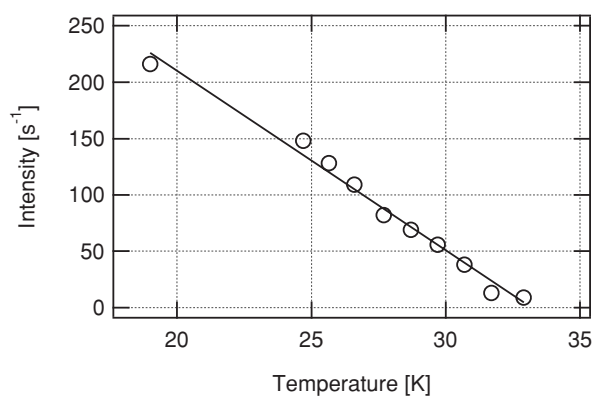

FIG. 13. Luminescence intensity versus the matrix temperature during the collision of $\mathrm{Ag}_{2}^{+}$. At $34 \mathrm{~K}$ the evaporation of the matrix sets in. The experiment is conducted under steady argon gas deposition and with a deposition energy for $\mathrm{Ag}_{2}^{+}$of $30 \mathrm{eV}$. The line is a guide to the eye.

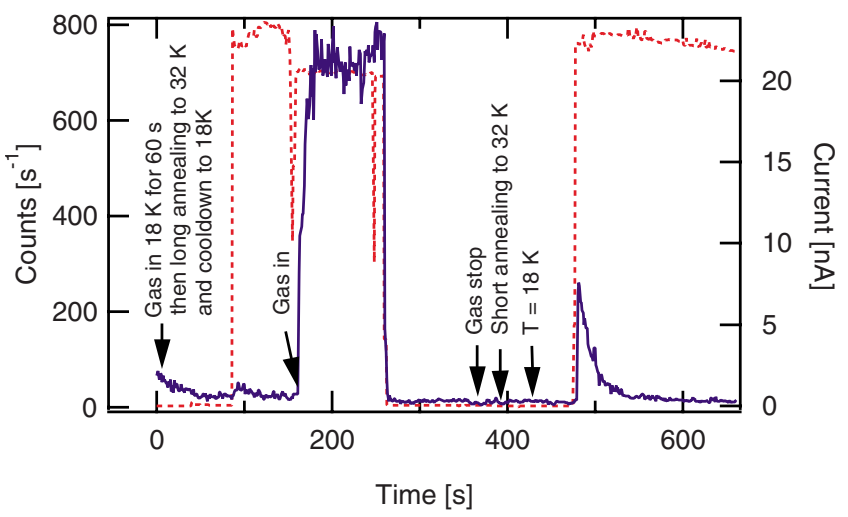

FIG. 14. (Color online) Matrix annealing experiments. Here again the continuous line shows the luminescence and the dotted line the measured current. A fresh matrix is first deposited without clusters at $18 \mathrm{~K}$ for $60 \mathrm{~s}$, it is then annealed by heating it to $32 \mathrm{~K}$ for $\sim 30 \mathrm{~s}$ and cooled down again to $18 \mathrm{~K}$; when the cluster current is switched on almost no luminescence is seen. Argon is then let into the system; this provides a reference for the luminescence intensity at $18 \mathrm{~K}$. Later the same experiment is renewed but this time with a short annealing cycle (heat and cool) the luminescence intensity is smaller due to incomplete annealing of the matrix.

$2 / 3$ of the sublimation temperature, fcc grains of about $100 \mathrm{~nm}$ are obtained, while when this temperature is lower than about $1 / 3$ of the sublimation temperature the grain size is about $10 \mathrm{~nm}$ and a higher density of packing defects is observed.

From the temperature dependence of the luminescence and the annealing per impact measurements shown above, we deduce that the structure of the matrix, in particular the amount of defects is a critical parameter that governs the intensity of the luminescence. This is further confirmed by the experiments shown in Fig. 14, where nearly no luminescence is observed from a cold but previously annealed matrix. Please note that the neutralization current is still measured under all conditions as it should be the case following the discussion above.

\section{A. Rare gases, solids, free excitons, and luminescence from impurities}

In Sec. III, we showed that collisions of ions on a rare gas matrix can produce electronic excitations in the matrix. An excitation above the energy gap of the rare gas solid results in electron-hole pair (free exciton) formation. Excitons and energy transfer mechanisms between excitons and impurities (the metal atoms and clusters in this case) have been studied thoroughly. ${ }^{25,26,28,47-53}$ In these studies, the excitons have been excited either by x-ray absorption, resonant excitation with synchrotron light, or electron bombardment. Exciton production by cluster ion collisions has, to our knowledge, not been studied.

The central point in the discussion derives from comparing our results to experiments by Schrimpf et al. ${ }^{51}$ who performed fluorescence measurements on atomic $\mathrm{Ag}$ and $\mathrm{Au}$ as well as on molecular oxygen by exciting with monochromatized synchrotron light. They were therefore able to excite 
the metal atoms resonantly by inner shell excitations or by producing excitons in the rare gas matrix. They show that the energy transfer from excitons to the metallic impurities leads to fluorescence of the impurities in the host matrix.

The relative peak intensities in the luminescence resulting from the collision of $\mathrm{Ag}_{1}^{+}$with the argon matrix (Fig. 7) is strikingly similar to their observations on exciton excitations for silver atoms. Moreover the presence of additional peaks in the luminescence of $\mathrm{Au}_{1}$ in $\mathrm{Ar}$ is very similar to the additional peak at $2.45 \mathrm{eV}$ that they observe for $\mathrm{Au}_{1}$ in $\mathrm{Kr}$ and tentatively attributed to $\mathrm{Au}-\mathrm{H}$ complexes in that paper. ${ }^{60} \mathrm{Fi}-$ nally the temperature dependence of the luminescence (Fig. 13) is in good agreement with their observation in $\mathrm{Kr}$ matrices.

The comparison provides experimental evidence that the excited states from which the luminescence proceeds is identical in both experiments. We therefore attribute the luminescence in our experiments to energy transfer from excitons to the metallic impurities.

Free excitons (FEs) produced in the collision of ions with the rare gas matrix can diffuse through the solid. ${ }^{50,52}$ Because of strong interaction with acoustic phonons the hole gets self-trapped rapidly. ${ }^{25}$ The diffusion length of FE depends strongly on the matrix preparation, being very short $(10-20 \mathrm{~nm})$ in polycrystalline matrices ${ }^{46,54}$ and extending to several hundreds $\mathrm{nm}$ in well annealed matrices at low temperature. ${ }^{55}$

Self-trapped holes are stable as long as the electron is trapped either at a structural defect of the matrix, a surface site or at a guest with positive electron affinity. ${ }^{28}$ In polycrystalline matrices, those electron traps have been observed. ${ }^{47,56}$ If the electron does not find a trap it recombines with the trapped hole. The energy is released by the emission of a VUV photon ${ }^{25,28}$ that has $\sim 50 \%$ chances to be captured by the metal surface and to induce a photoelectron into the conduction band of the rare gas as discussed in Sec. III. This explains the observed temperature dependence of the luminescence (Fig. 13) and gives hints about the microscopic mechanism involved in the luminescence process.

\section{B. Microscopic model for the luminescence process}

In pure and doped rare gas solids there have been several studies of thermally stimulated luminescence (TSL), conduction (TSC), and exoelectron emission (TSEE) (Refs. 47-49, 56, and 57) by different groups. The solid matrices are activated at low temperature by an electron beam or by synchrotron radiation; later the temperature is raised slowly and the luminescence, the conduction or the electron emission is recorded simultaneously. These three processes can all be linked to the release of the electrons from the structural defects or impurity traps. We believe this luminescence process is the same in nature as the one we observe. As mentioned above, electrons are trapped at structural defects which are annealed by the local annealing induced by the impact of the cluster on the matrix. When this happens, the electron is released and can recombine with a self-trapped hole, ${ }^{56,57}$ the energy is dissipated either by the emission of a VUV photon or by exciting a closeby impurity, in our case a metal cluster, via an energy exchange mechanism. ${ }^{25,52}$

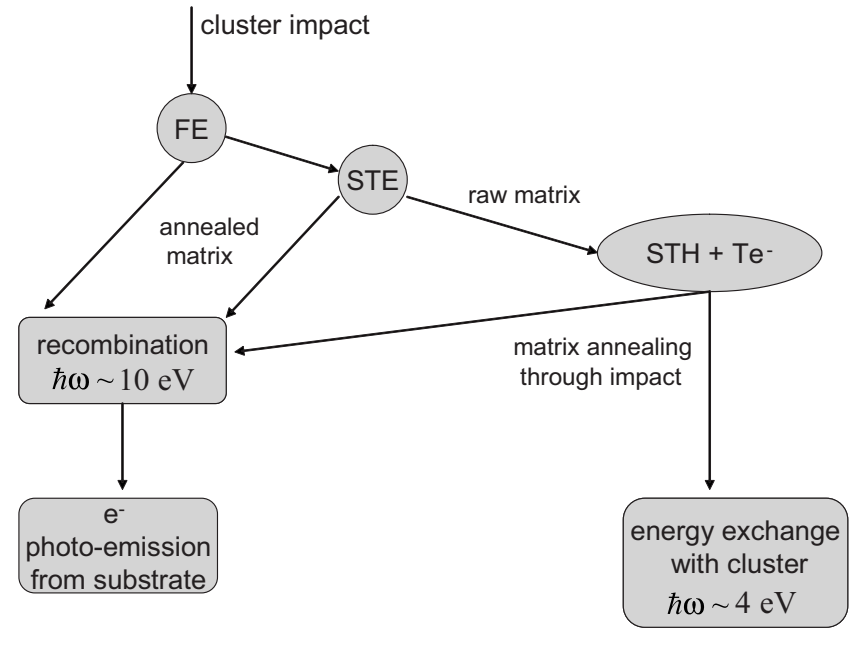

neutralization

luminescence

FIG. 15. Free excitons (FE) are created in the collision process. Due to the strong interaction with the matrix deposited at low temperature most of FE become self-trapped excitons (STE). When the STE recombine a high energy photon is emitted that has a $50 \%$ probability to be absorbed by the close-by metal substrate and produce a photoelectron responsible for the neutralization. When the matrix is grown at low temperature the trapping of the electron on defects results in two stable entities the self-trapped hole (STH) and the trapped electron $\left(\mathrm{Te}^{-}\right)$. As long as the electron is trapped the excitation is stable. This is the initial situation in the thermally stimulated experiments (TSL, TSC, TSEE, see text). In our experiment the electron is liberated by the local annealing resulting from the collision process. Due to the close proximity with the cluster an energy exchange mechanism between the STE and the atom or cluster can take place and result in the observed fluorescence.

In detail, the luminescence mechanism requires several steps (Fig. 15). (1) The collision with the matrix first produces a free exciton (FE). (2) The strong interaction of the hole with the rough matrix causes it to self-trap within a distance of $\sim 20 \mathrm{~nm}$ (self-trapped exciton STE). (3) When the matrix is grown at low temperature, structural defects are present; the electron can be trapped at a defect site. We have here a self-trapped hole and a trapped electron $\left(\mathrm{STH}+\mathrm{Te}^{-}\right)$, which is stable as the electron is trapped. (4) The cluster impact induces a local annealing of the matrix. The time associated with this annealing mechanism is at least 10 to 50 ps. $^{15}$ (5) The exciton energy is released because the electron is freed by the local annealing of the matrix. An energy transfer mechanism between the exciton and a nearby cluster causes the luminescence of this cluster.

It is interesting to note that according to this model the luminescence and the production of electrons for the neutralization are separate processes. While the emission of a VUV photon can happen at any temperature the trapping of the free exciton associated with the luminescence requires the presence of structural defects in the matrix which are temperature dependent. This is in agreement with the experimental observations.

In the mechanism discussed above, the source of the molecular self-trapped hole (the number of free excitons trapped 
in the proximity of the surface of the matrix) depends on temperature via the crystallinity of the matrix. In thermally stimulated processes such as TSL, TSEE, or TSC, in contrast, this is a drain term for these self-trapped holes (the number of trapped electrons released) that are thermally activated. For that reason Fig. 13 carries information about the trapping of the exciton/electron as a function of temperature) and it is therefore not possible to compare our measurements to the rates versus temperature for TSL or TSEE, even though the mechanism is the same. Actually the temperature dependence reported in Fig. 13 agrees with the dependence in trapping distance with the crystallinity of the matrix discussed above. Note that the 10-20 nm diffusion length measured by Herkert et al. ${ }^{52}$ for low-energy FE is also in excellent agreement with the $20 \mathrm{~nm}$ active layer that we report. This active layer was found to be independent on the impact energy of the particle, also in agreement with the model exposed here.

\section{CONCLUSION}

The electronic excitations during the collision of ions with rare gas matrices on a metal substrate are studied experimentally through the luminescence of the projectile. A convergent set of experimental evidence together with data known from the literature show that free excitons are created in the collision process. The recombination of the excitons produce VUV photons that explain the neutralization of the deposited particles in the matrix. The luminescence itself is due to molecular self-trapped holes close to the matrix-vacuum interface that recombine when electrons are liberated by the matrix annealing due to the collision.
${ }^{1}$ S. Fedrigo, W. Harbich, and J. Buttet, Phys. Rev. B 47, 10706 (1993).

${ }^{2}$ E. C. Honea, A. Ogura, C. A. Murray, K. Raghavachari, W. O. Sprenger, M. F. Jarrold, and W. L. Brown, Nature (London) 366, 42 (1993).

${ }^{3}$ G. Vandoni, C. Félix, R. Monot, J. Buttet, and W. Harbich, Chem. Phys. Lett. 229, 51 (1994).

${ }^{4}$ K. Bromann, C. Félix, H. Brune, W. Harbich, R. Monot, J. Buttet, and K. Kern, Science 274, 956 (1996).

${ }^{5}$ A. Sanchez, S. Abbet, U. Heiz, W.-D. Schneider, H. Häkkinen, R. N. Barnett, and U. Landman, J. Phys. Chem. 103, 9573 (1999).

${ }^{6}$ R. Schaub, H. Jödicke, F. Brunet, R. Monot, J. Buttet, and W. Harbich, Phys. Rev. Lett. 86, 3590 (2001).

${ }^{7}$ C. Félix, C. Sieber, W. Harbich, J. Buttet, I. Rabin, W. Schulze, and G. Ertl, Phys. Rev. Lett. 86, 2992 (2001).

${ }^{8}$ J. T. Lau, A. Fohlisch, R. Nietubyc, M. Reif, and W. Wurth, Phys. Rev. Lett. 89, 057201 (2002).

${ }^{9}$ R. Palmer, S. Pratontep, and H.-G. Boyen, Nat. Mater. 2, 443 (2003).

${ }^{10}$ J.-M. Antonietti, M. Michalski, U. Heiz, H. Jones, K. H. Lim, N. Rösch, A. Del Vitto, and G. Pacchioni, Phys. Rev. Lett. 94, 213402 (2005).

${ }^{11}$ S. Serrano-Guisan, G. Di Domenicantonio, M. Abid, J.-P. Abid, M. Hillenkamp, L. Gravier, J.-P. Ansermet, and C. Félix, Nat. Mater. 5, 730 (2006).

${ }^{12}$ H. P. Cheng and U. Landman, Science 260, 1304 (1993).

${ }^{13}$ G. Vandoni, C. Félix, and C. Massobrio, Phys. Rev. B 54, 1553 (1996).

${ }^{14}$ C. Massobrio, B. Nacer, T. Bekkay, G. Vandoni, and C. Félix, Surf. Sci. 385, 87 (1997).

${ }^{15}$ M. Ratner, W. Harbich, and S. Fedrigo, Phys. Rev. B 60, 11730 (1999).

${ }^{16} \mathrm{Bu}$. Wrenger, K. H. Meiwes-Broer, O. Speer, and M. E. Garcia, Phys. Rev. Lett. 79, 2562 (1997).

${ }^{17}$ N. Lorente, R. Monreal, M. Alducin, and P. Apell, Z. Phys. D: At., Mol. Clusters 41, 143 (1997).

${ }^{18}$ E. B. Gordon, V. V. Khmelenko, and O. S. Rzhevsky, Chem. Phys. Lett. 217, 605 (1994).

${ }^{19}$ A. Usenko, G. Frossati, and E. B. Gordon, Phys. Rev. Lett. 90,
$153201(2003)$

${ }^{20}$ S. Fedrigo, W. Harbich, and J. Buttet, J. Chem. Phys. 99, 5712 (1993).

${ }^{21}$ C. Félix, C. Sieber, W. Harbich, J. Buttet, I. Rabin, W. Schulze, and G. Ertl, Chem. Phys. Lett. 313, 105 (1999).

${ }^{22}$ C. Sieber, J. Buttet, W. Harbich, C. Félix, R. Mitrić, and V. Bonačić-Koutecký, Phys. Rev. A 70, 041201(R) (2004).

${ }^{23}$ C. Sieber, W. Harbich, K.-H. Meiwes-Broer, and C. Félix, Chem. Phys. Lett. 433, 32 (2006)

${ }^{24}$ F. Conus, J. T. Lau, V. Rodrigues, and C. Félix, Rev. Sci. Instrum. 77, 113103 (2006).

${ }^{25}$ N. Schwentner, E.-E. Koch, and J. Jortner, Electronic Excitations in Condensed Rare Gases, Vol. 107 of Springer Tracts in Modern Physics (Springer, Berlin, 1985).

${ }^{26}$ G. Zimmerer, in Excited-State Spectroscopy in Solids (North Holland, Amsterdam, 1987), pp. 37-110.

${ }^{27}$ I. Y. Fugol', Adv. Phys. 37, 1 (1988).

${ }^{28}$ K. S. Song and R. T. Williams, Self-Trapped Excitons, Vol. 105 of Springer Series in Solid State Science (Springer, Berlin, 1996).

${ }^{29}$ R. Johnson and J. Schou, in Fundamental Processes in The Sputtering of Atoms and Molecules, Vol. 43 Matematisk-fysiske meddelelser, edited by P. Sigmund (Royal Danish Society, Copenhagen, 1993), pp. 403-494.

${ }^{30}$ D. J. O'Shaughnessy, J. W. Boring, S. Cui, and R. E. Johnson, Phys. Rev. Lett. 61, 1635 (1988).

${ }^{31}$ B. Van Zyl, H. Neumann, H. L. Rothwell, Jr., and R. C. Amme, Phys. Rev. A 21, 716 (1980).

${ }^{32}$ B. Van Zyl, H. L. Rothwell, Jr., and H. Neumann, Phys. Rev. A 21, 730 (1980).

${ }^{33}$ B. Van Zyl, M. W. Gealy, and H. Neumann, Phys. Rev. A 35, 4551 (1987).

${ }^{34}$ B. Van Zyl and M. W. Gealy, Phys. Rev. A 35, 3741 (1987).

${ }^{35}$ P. O. Haugsjaa, R. C. Amme, and N. G. Utterback, Phys. Rev. Lett. 22, 322 (1969).

${ }^{36}$ P. O. Haugsjaa and R. C. Amme, Phys. Rev. Lett. 23, 633 (1969).

${ }^{37}$ J. Rothwell, H. L., R. C. Amme, and B. Van Zyl, Phys. Rev. Lett. 36, 785 (1976).

${ }^{38}$ R. C. Amme and P. O. Haugsjaa, Phys. Rev. 177, 230 (1969).

${ }^{39}$ D. H. Waldeck, A. P. Alivisatos, and C. B. Harris, Surf. Sci. 158, 
103 (1985).

${ }^{40}$ R. R. Chance, A. Prock, and R. Silbey, J. Chem. Phys. 60, 2744 (1974).

${ }^{41}$ S. Fedrigo, W. Harbich, and J. Buttet, Int. J. Mod. Phys. B 6, 23 (1992).

${ }^{42}$ G. A. Ozin and S. A. Mitchell, J. Phys. Chem. 86, 473 (1982).

${ }^{43}$ I. Rabin, W. Schulze, and G. Ertl, Chem. Phys. Lett. 312, 5 (1999).

${ }^{44}$ W. Harbich, S. Fedrigo, and J. Buttet, Z. Phys. D: At., Mol. Clusters 26, 138 (1993).

${ }^{45}$ J. A. Venables and B. L. Smith, in Crystal Growth and Crystal Defects, edited by M. L. Klein and J. A. Venables (Academic Press, London, 1977), Vol. Rare Gas Solids II, Chap. 10, pp. 609-662.

${ }^{46}$ I. Y. Fugol', Adv. Phys. 27, 1 (1978).

${ }^{47}$ A. Schrimpf, C. Boekstiegel, H.-J. Stöckmann, T. Bornemann, K. Ibbeken, J. Kraft, and B. Herkert, J. Phys.: Condens. Matter 8, 3677 (1996).

${ }^{48}$ J. Becker, O. N. Grigorashchenko, A. N. Ogurtsov, M. Runne, E. V. Savchenko, and G. Zimmerer, J. Phys. D 31, 749 (1998).

${ }^{49}$ E. V. Savchenko, O. N. Grigorashchenko, A. N. Ogurtsov, V. V. Rudenkov, G. B. Gumenchuk, M. Lorenz, A. M. SmithGicklhorn, M. Frankowski, and V. E. Bondybey, Surf. Rev. Lett. 9, 353 (2002).

${ }^{50}$ Z. Ophir, N. Schwentner, B. Raz, M. Skibowski, and J. Jortner, J. Chem. Phys. 63, 1072 (1975).

${ }^{51}$ A. Schrimpf, B. Herkert, L. Manceron, U. Schriever, and H.-J.
Stöckmann, Phys. Status Solidi B 165, 469 (1991).

${ }^{52}$ B. Herkert, A. Schrimpf, K. Göttsche, T. Bornemann, and H.-J. Stöckmann, Phys. Rev. B 51, 15763 (1995).

${ }^{53}$ M. Lengen, M. Joppien, R. von Pietrowski, and T. Möller, Chem. Phys. Lett. 229, 362 (1994).

${ }^{54}$ E. Roick, R. Gaethke, G. Zimmerer, and P. Gurtler, Solid State Commun. 47, 333 (1983).

${ }^{55}$ D. Varding, J. Becker, L. Frankenstein, B. Peters, M. Runne, A. Schroder, and G. Zimmerer, Low Temp. Phys. 19, 427 (1993).

${ }^{56}$ E. Savchenko, O. Grigorashchenko, A. Ogurtsov, V. Rudenkov, G. Gumenchuk, M. Lorenz, M. Frankowski, A. SmithGicklhorn, and V. Bondybey, Surf. Sci. 507-510, 754 (2002).

${ }^{57}$ R. Dersch, B. Herkert, M. Witt, H.-J. Stöckmann, and H. Ackermann, Z. Phys. B: Condens. Matter 80, 39 (1990).

${ }^{58}$ Electrons accelerated by the electrical field are energetic and do not necessarily neutralize a positive charge, but will be only deflected by it.

${ }^{59} \mathrm{~A}$ definite proof for that would be to look for VUV luminescence signature of the recombination of free or trapped excitons. This has not been done for technical reasons.

${ }^{60}$ The peak at $2.45 \mathrm{eV}$ is most probably due to a long lived state that is excited with excitons but that cannot be populated by UV-Vis optical excitation. The suggested assignment to $\mathrm{AuH}_{x}$ complexes is probably wrong as well as the comparison with the fluorescence of $\mathrm{Ag}_{3}$ suggested in Ref. 52. We see no reasons for an hydrogen contamination in the present experiments. 